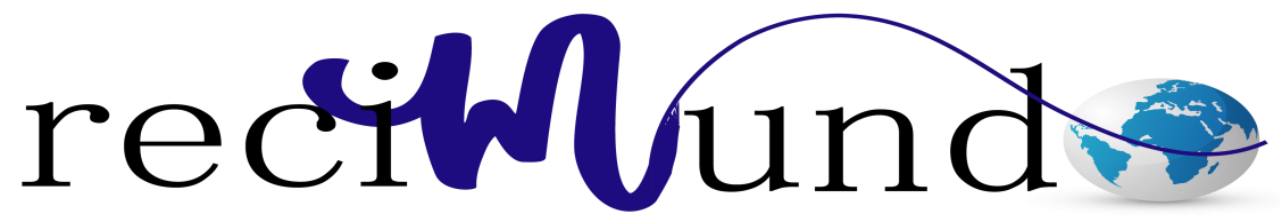

Revista Científica Mundo de la Investigación y el Conocimiento

Glubis Gomez Pelaez ${ }^{\text {a }}$ Carlos Lara Zambrano ${ }^{\text {b; Hugo Luna Rodriguez }}{ }^{\text {c }}$; Carlos Lara Parra ${ }^{\mathrm{d}}$

Factores de riesgo de la Criptococosis en pacientes con VIH en el hospital de infectología “Dr. José Daniel Rodriguez Maridueña” en el periodo 2015-2016

Revista Científica Mundo de la Investigación y el Conocimiento. Vol. 2 núm., 1, febrero, ISSN: 2588-073X, 2018, pp. 157-171

DOI: $10.26820 /$ recimundo/2.1.2018.157-171

Editorial Saberes del Conocimiento

Recibido: 05/12/2017

Aceptado: 10/02/2018 
Factores de riesgo de la Criptococosis en pacientes con VIH en el hospital de infectología "Dr. José Daniel Rodriguez Maridueña" en el periodo 2015-2016 Vol. 2, núm. 1., (2018)

Glubis Gomez Pelaez; Carlos Lara Zambrano; Hugo Luna Rodriguez; Carlos Lara Parra

\section{RESUMEN}

Antecedentes: La criptococosis es una infección fúngica sistémica que afecta fundamentalmente a pacientes inmunocomprometidos. La incidencia de la infección presentó un aumento aparejado al de la epidemia del síndrome de inmunodeficiencia adquirida (sida) desde su inicio en la década de 1980. La criptococosis se considera una de las micosis oportunistas más importantes en los últimos tiempos; a pesar de esto, en Ecuador son pocos los reportes relacionados con la misma. Métodos: El presente estudio tiene un enfoque cuantitativo de diseño no experimental, de corte transversal y retrospectivo con la utilización del método de observación analítica, cuyo objetivo es proveer información sobre los factores de riesgo de la Criptococosis en personas con VIH. Se revisaron 115 historias clínicas de pacientes con VIH que presentaron Criptococosis y fueron ingresados en el Hospital Dr. José Daniel Rodríguez Maridueña, durante el periodo 20152016. Resultados: Se evidenció que la concentración sanguínea menor de 200 CD4 cel/mm3 constituyó el factor más preponderante para la manifestación de la criptococosis, además se pudo constatar que la infección por criptococo se presentó mayormente en la población que habita en áreas Rurales.

Palabras clave: VIH, criptococosis, factores de riesgo. 


\title{
Factores de riesgo de la Criptococosis en pacientes con VIH en el hospital de infectología “Dr. José Daniel Rodriguez Maridueña” en el periodo 2015-2016
}

Vol. 2, núm. 1., (2018)

Glubis Gomez Pelaez; Carlos Lara Zambrano; Hugo Luna Rodriguez; Carlos Lara Parra

\begin{abstract}
Background: Cryptococcosis is a systemic fungal infection that primarily affects immunocompromised patients. The incidence of infection increased as a result of the epidemic of acquired immunodeficiency syndrome (AIDS) since its inception in the 1980s. Cryptococcosis is considered one of the most important opportunistic mycoses in recent times; Despite this, in Ecuador there are few reports related to it. Methods: The present study has a quantitative approach of non-experimental cross-sectional and retrospective design with the use of the analytical observation method for a wide information on the subject, whose objective is to provide information on the risk factors of Cryptococcosis in People with HIV. We reviewed 115 medical records of patients with HIV who presented Cryptococcosis and were admitted to the Dr. José Daniel Rodríguez Maridueña Hospital during the period 2015-2016. Results: It was evidenced that the blood concentration of less than $200 \mathrm{CD} 4$ cells / $\mathrm{mm} 3$ was the most important factor for the manifestation of cryptococcosis, and it was possible to verify that cryptococcal infection was present mainly in the population living in rural areas.
\end{abstract}

Keywords: HIV, cryptococcosis, risk factors. 


\section{Factores de riesgo de la Criptococosis en pacientes con VIH en el hospital de infectología "Dr. José Daniel Rodriguez Maridueña" en el periodo 2015-2016}

Vol. 2, núm. 1., (2018)

Glubis Gomez Pelaez; Carlos Lara Zambrano; Hugo Luna Rodriguez; Carlos Lara Parra

\section{Introducción.}

La criptococosis es una infección fúngica sistémica que afecta, fundamentalmente, a pacientes inmunocomprometidos. La misma resulta de la inhalación de esporas del complejo de especies Cryptococcus neoformans para el cual se reconocen, en la actualidad, siete especies de vida libre en la naturaleza. Estas levaduras presentan especial tropismo por el sistema nervioso central. Esto justifica que entre el 70 y $90 \%$ de los pacientes manifiesten cuadros clínicos de meningitis o meningoencefalitis subaguda; no obstante, sus síntomas suelen ser inespecíficos. Según los reportes, ocasiona mortalidad en el $60 \%$ de las personas afectadas a pesar del tratamiento antifúngico convencional y puede alcanzar el $100 \%$ en los pacientes no tratados o con tratamientos iniciados tardíamente ${ }^{1-6}$.

La incidencia de la infección presentó un aumento aparejado al de la epidemia del síndrome de inmunodeficiencia adquirida (sida) desde su inicio en la década de 1980.5 Adicionalmente, otras condiciones tales como los tratamientos inmunosupresores y los trasplantes, también son consideradas factores predisponentes para el desarrollo de la enfermedad. La criptococosis se considera una de las micosis oportunistas más importantes en los últimos tiempos; a pesar de esto, en Ecuador son pocos los reportes relacionados con la $\operatorname{misma}^{6-10}$.

El propósito de este trabajo de investigación es el estudio de los factores de riesgo y características de la Criptococosis en 115 pacientes con VIH atendidos en el Hospital de Infectología Dr. José Daniel Rodríguez Maridueña, durante el periodo 2015-2016, a través de un análisis profundo de las historias clínicas que permitan ver la evolución de los pacientes y el 


\section{Factores de riesgo de la Criptococosis en pacientes con VIH en el hospital de infectología “Dr. José Daniel Rodriguez Maridueña” en el periodo 2015-2016}

Vol. 2, núm. 1., (2018)

Glubis Gomez Pelaez; Carlos Lara Zambrano; Hugo Luna Rodriguez; Carlos Lara Parra desarrollo de las manifestaciones clínicas mediante un estudio observacional, el cual se evaluara sus complicaciones, sintomatología, cuadro clínico, diagnóstico y tratamiento, este trabajo cumplirá el objetivo de informar acerca de esta enfermedad oportunista común en pacientes con VIH a nivel local ${ }^{11-12}$.

\section{Metodología.}

El presente estudio de investigación tiene un enfoque cuantitativo de diseño no experimental, de corte transversal y retrospectivo con la utilización del método de observación analítica, para una amplia información sobre el tema, cuyo objetivo es proveer información sobre los factores de riesgo de la Criptococosis en personas con VIH.

Se revisaron 115 historias clínicas de pacientes con VIH que presentaron Criptococosis y fueron ingresados en el Hospital Dr. José Daniel Rodríguez Maridueña, durante el periodo 20152016.

Universo y muestra

Corresponde a los 115 pacientes con VIH que presentaron Criptococosis y fueron ingresados en el Hospital Dr. José Daniel Rodríguez Maridueña, durante el periodo 2015-2016.

\section{Viabilidad}

Este trabajo de investigación es un estudio viable porque tiene la aprobación del Director del Hospital de Infectología Dr. José Daniel Rodríguez Maridueña, de los representantes de la Universidad de Guayaquil para su ejecución, que permitieron el acceso a la evaluación del estado 
Factores de riesgo de la Criptococosis en pacientes con VIH en el hospital de infectología "Dr. José Daniel Rodriguez Maridueña” en el periodo 2015-2016 Vol. 2, núm. 1., (2018)

Glubis Gomez Pelaez; Carlos Lara Zambrano; Hugo Luna Rodriguez; Carlos Lara Parra

de los pacientes VIH. Es de interés para un grupo de médicos especialistas del área de Infectología por ser casos muy frecuentes en la institución, para que existan datos estadísticos que demuestren la prevalencia de la Criptococosis, se permitirá estudiar los factores de riesgo de la Criptococosis del paciente con VIH, sus complicaciones, su diagnóstico, sintomatología y tratamiento que se les brindo. Este trabajo es autofinanciado por el autor.

\section{Resultados.}

Casos reportados con VIH+ criptococosis en años 2015-2016.

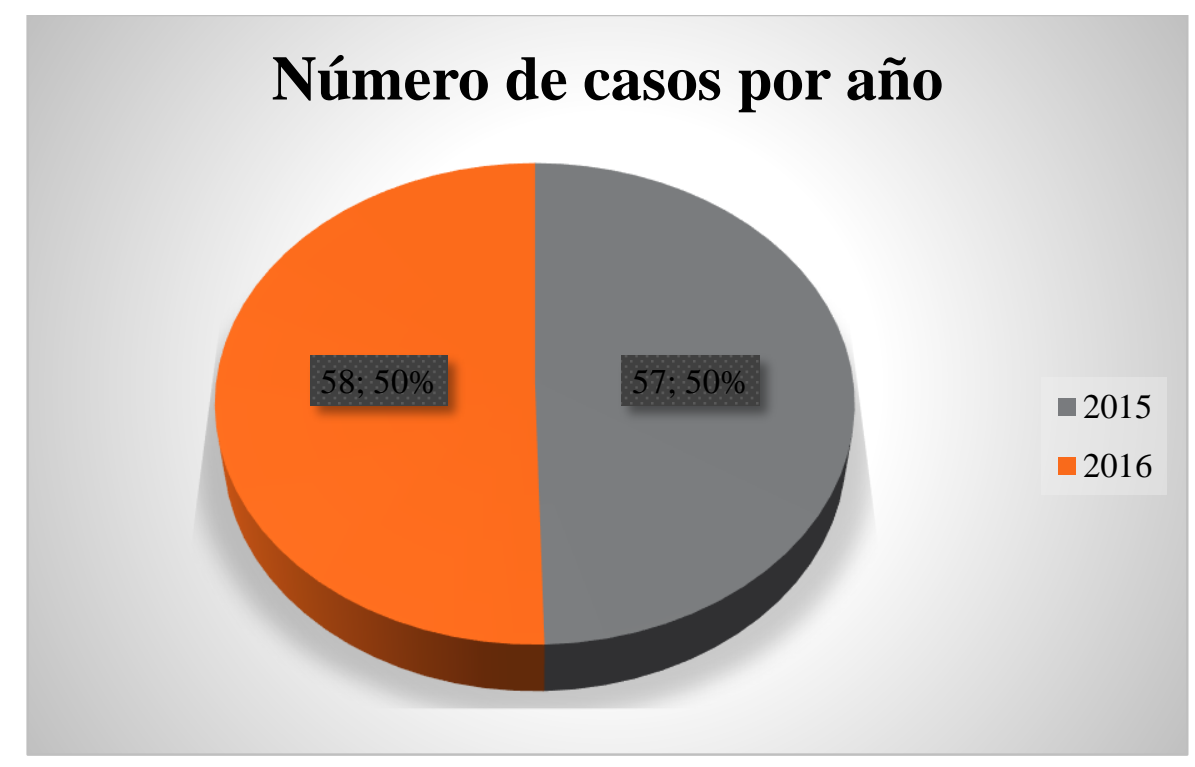

Figura $N^{\circ}$ 1.- Casos por año de criptococosis en el periodo 2015-2016

Interpretación: Número de casos confirmados de criptococosis en el periodo 2015-2016, la incidencia es similar en ambos años. 
Factores de riesgo de la Criptococosis en pacientes con VIH en el hospital de infectología “Dr. José Daniel Rodriguez Maridueña” en el periodo 2015-2016 Vol. 2, núm. 1., (2018)

Glubis Gomez Pelaez; Carlos Lara Zambrano; Hugo Luna Rodriguez; Carlos Lara Parra

\section{Formas de presentaciones clínicas de la Criptococosis}

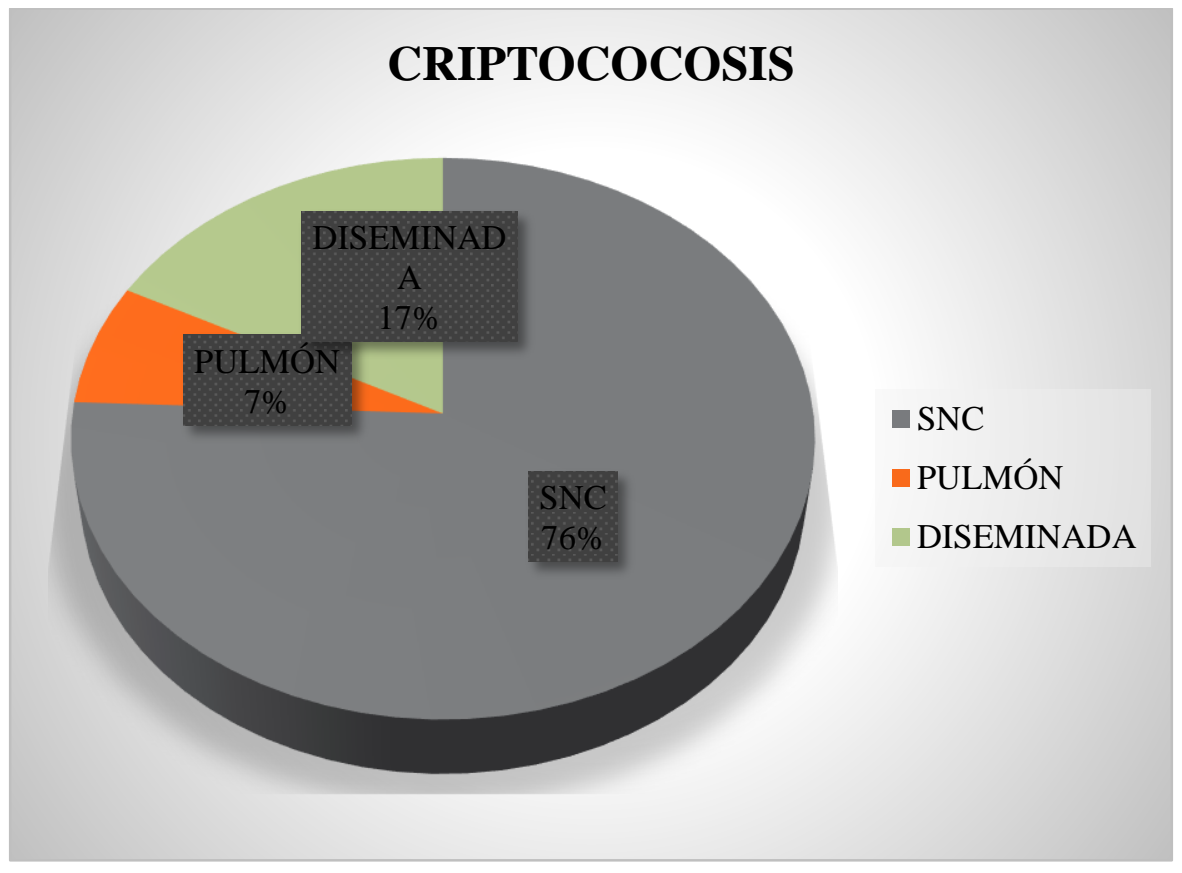

Figura $N^{\circ}$ 2.- Formas de presentación clínica de la criptococosis en el hospital de infectología

Interpretación: En el 76\% de casos, la criptococosis se manifestó en el sistema nervioso central demostrando el tropismo del hongo hacia esa zona del organismo, seguido de la presentación diseminada y pulmonar. 
Factores de riesgo de la Criptococosis en pacientes con VIH en el hospital de infectología "Dr. José Daniel Rodriguez Maridueña" en el periodo 2015-2016 Vol. 2, núm. 1., (2018)

Glubis Gomez Pelaez; Carlos Lara Zambrano; Hugo Luna Rodriguez; Carlos Lara Parra

Casos reportados con VIH-criptococosis de acuerdo al sexo

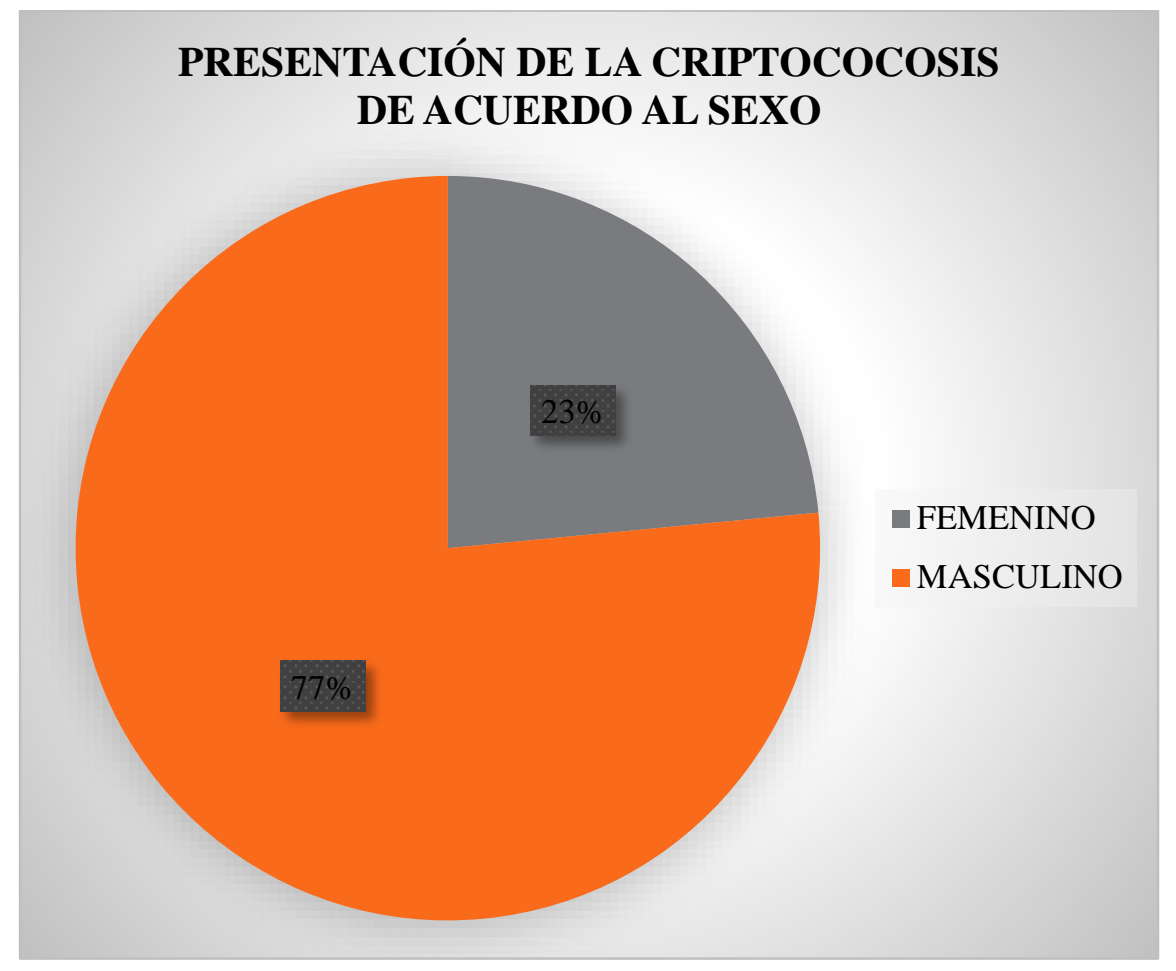

Figura $N^{\circ}$ 3.- Casos reportados con criptococosis clasificados de acuerdo al sexo

Interpretación: La criptococosis se presentó mayormente en el sexo masculino. 
Factores de riesgo de la Criptococosis en pacientes con VIH en el hospital de infectología “Dr. José Daniel Rodriguez Maridueña” en el periodo 2015-2016 Vol. 2, núm. 1., (2018)

Glubis Gomez Pelaez; Carlos Lara Zambrano; Hugo Luna Rodriguez; Carlos Lara Parra

Casos reportados con VIH-criptococosis de acuerdo a edad

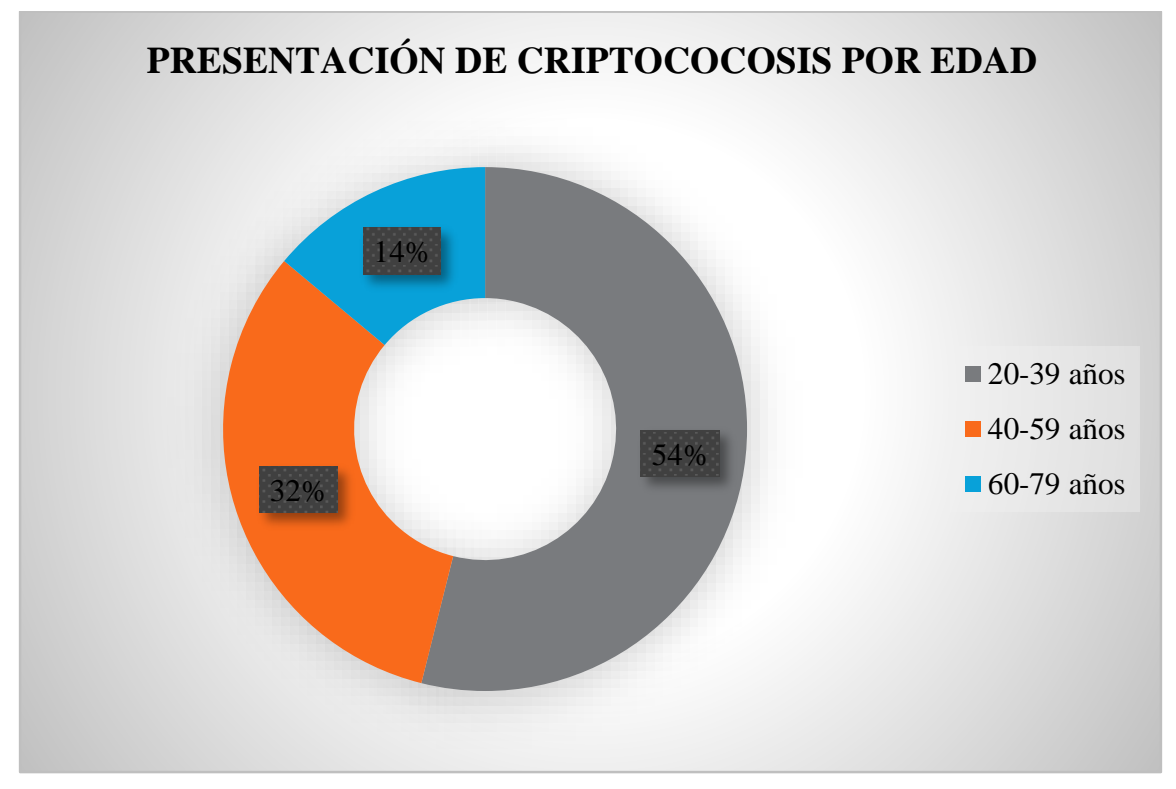

Figura $N^{\circ}$ 4.- Casos reportados con criptococosis clasificados de acuerdo a la edad

Interpretación: El grupo etario en el que se presentó mayormente la criptococosis fue entre los 20-39 años, con un 54\% de los casos. 
Factores de riesgo de la Criptococosis en pacientes con VIH en el hospital de infectología "Dr. José Daniel Rodriguez Maridueña" en el periodo 2015-2016 Vol. 2, núm. 1., (2018)

Glubis Gomez Pelaez; Carlos Lara Zambrano; Hugo Luna Rodriguez; Carlos Lara Parra

Zona de procedencia de los pacientes diagnosticados con VIH+criptococosis

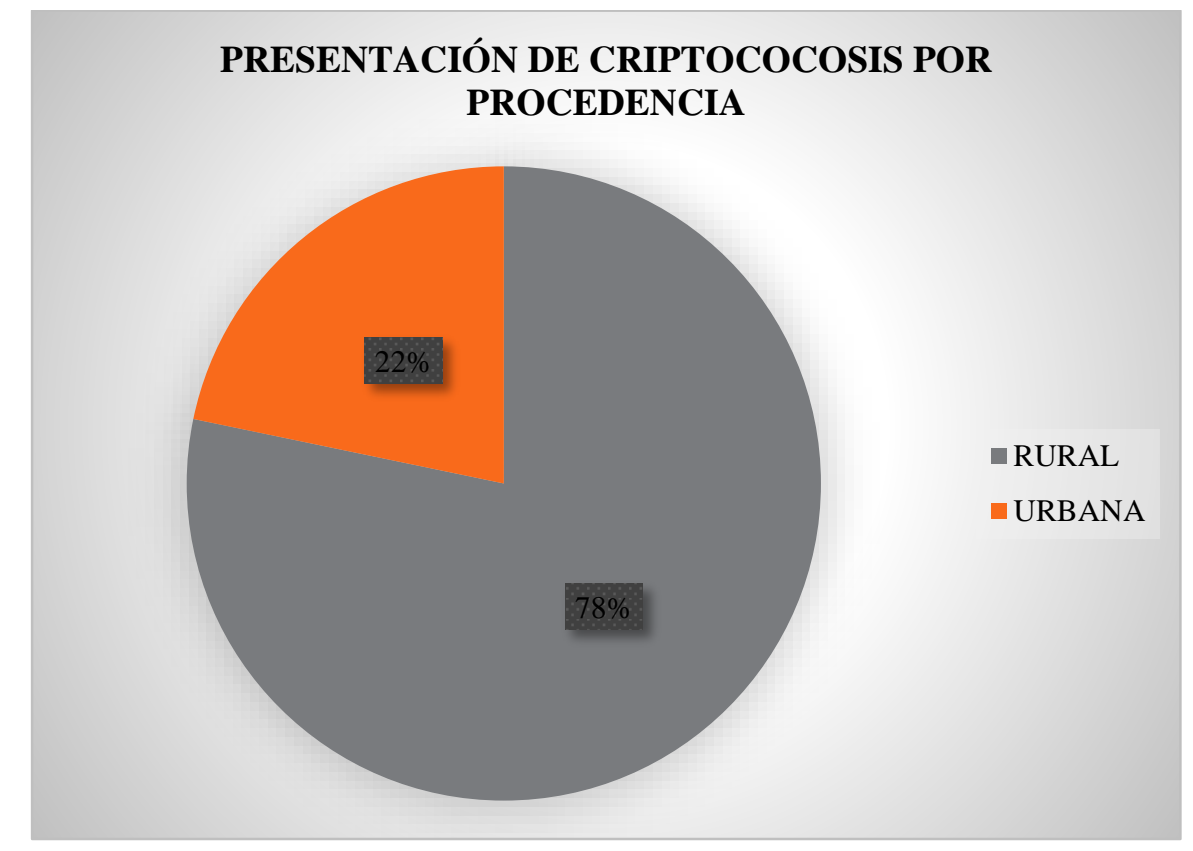

Figura $N^{\circ}$ 5.- Zona de procedencia de los pacientes diagnosticados con VIH+CRIPTOCOCOSIS

Interpretación: El porcentaje mayoritario de la criptococosis se presentó en la población rural con un $78 \%$, lo que indica el mayor contacto que tienen los habitantes de esta zona con las esporas del hongo. 
Factores de riesgo de la Criptococosis en pacientes con VIH en el hospital de infectología “Dr. José Daniel Rodriguez Maridueña” en el periodo 2015-2016 Vol. 2, núm. 1., (2018)

Glubis Gomez Pelaez; Carlos Lara Zambrano; Hugo Luna Rodriguez; Carlos Lara Parra

Concentración de células cd4 en sangre al momento del diagnóstico de VIH+criptococosis

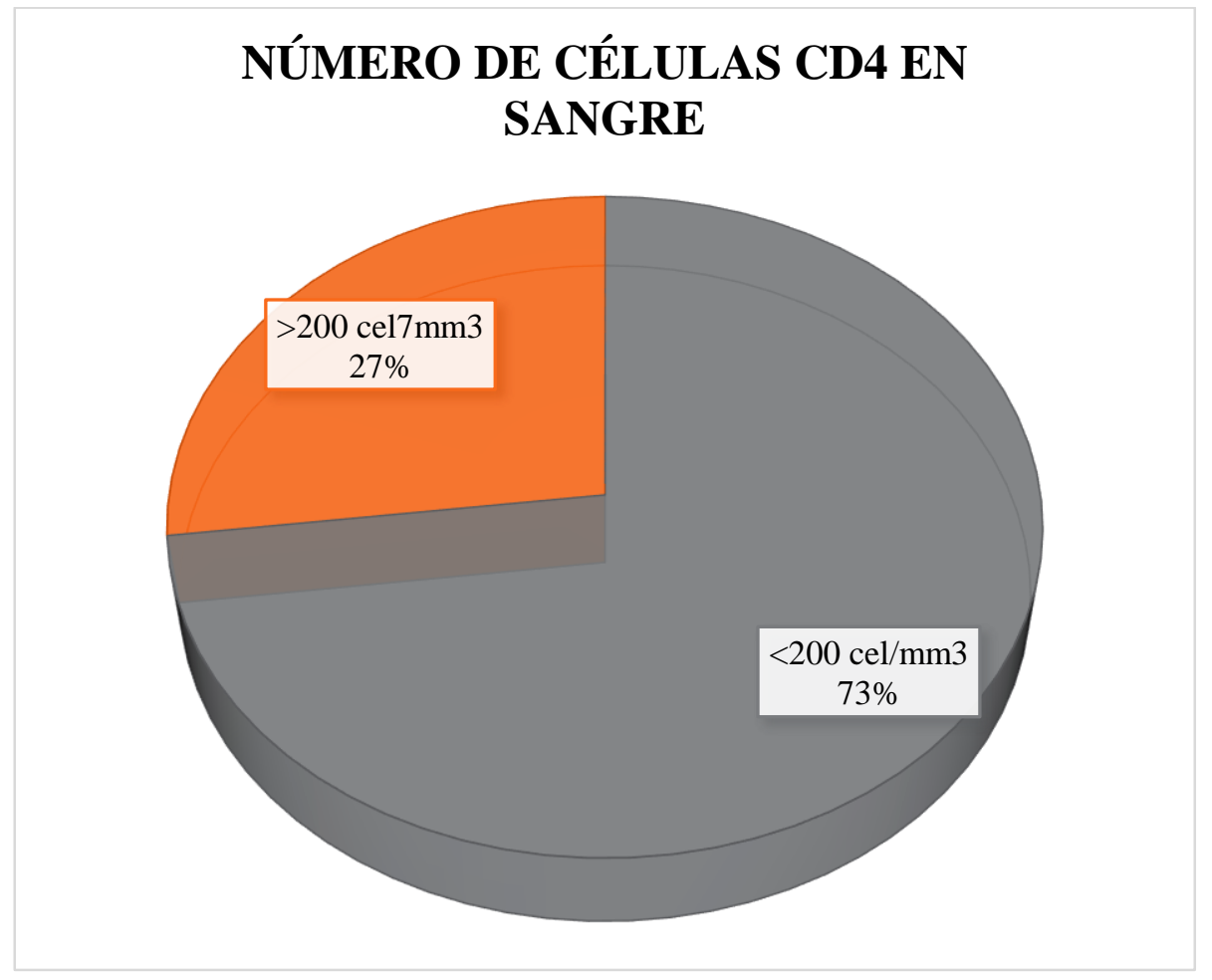

Figura $\mathbf{N}^{\circ}$ 6.- Concentración sanguínea de células al momento del diagnóstico de criptococosis

Interpretación: Siendo la criptococosis una enfermedad oportunista el número de células CD4 en sangre constituye un factor preponderante para la presentación de la misma, lo que se demuestra en este estudio, en el cual el $73 \%$ de pacientes tenía menos de 200cel $/ \mathrm{mm} 3$. 
Factores de riesgo de la Criptococosis en pacientes con VIH en el hospital de infectología "Dr. José Daniel Rodriguez Maridueña" en el periodo 2015-2016 Vol. 2, núm. 1., (2018)

Glubis Gomez Pelaez; Carlos Lara Zambrano; Hugo Luna Rodriguez; Carlos Lara Parra

Reporte de casos de VIH+ criptococosis de acuerdo a egreso hospitalario en el año 2015

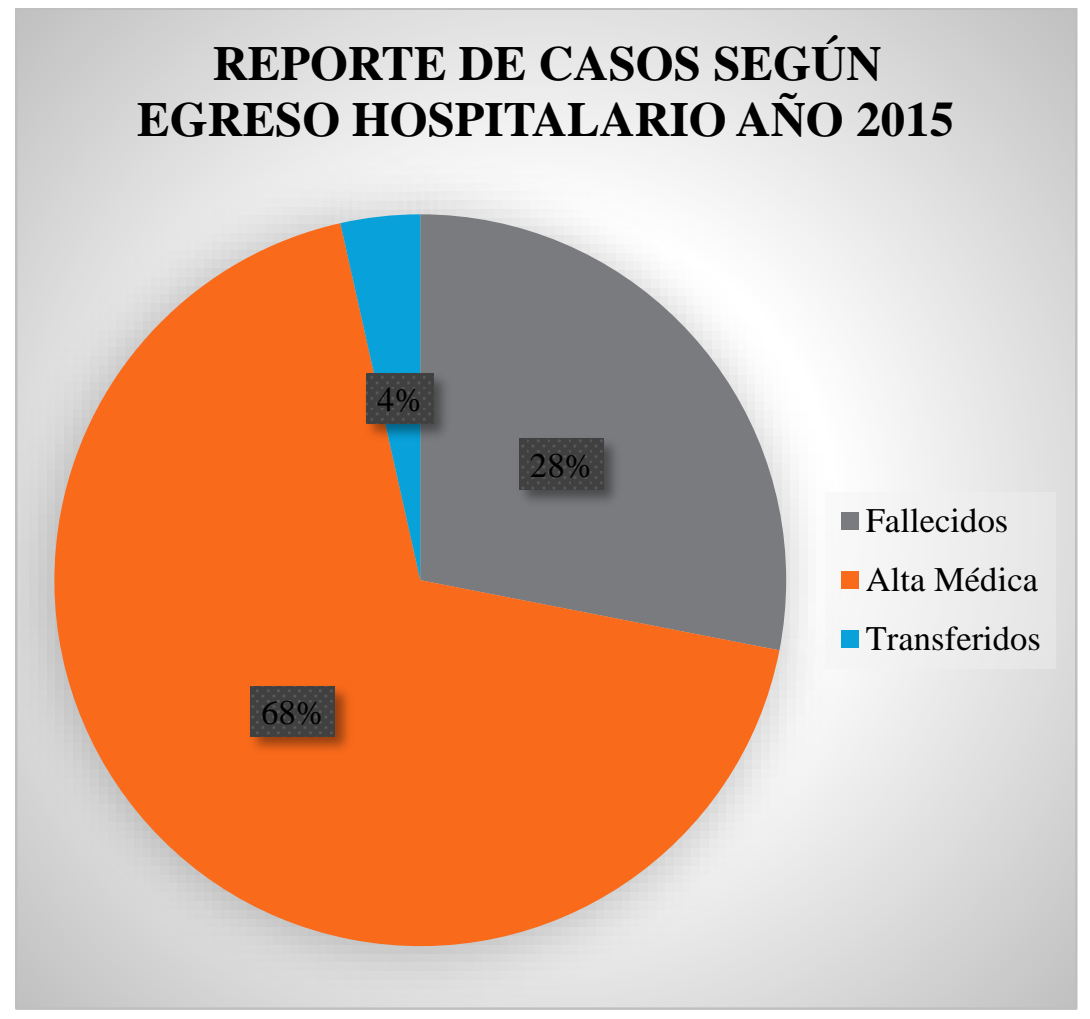

Figura $N^{\circ}$ 7.- Casos de VIH+ criptococosis de acuerdo a egreso hospitalario en el año 2015

Interpretación: En el año 2015 el 28\% de pacientes con Vih+criptococosis que fue atendido en el hospital de infectología falleció debido a sus complicaciones. 
Factores de riesgo de la Criptococosis en pacientes con VIH en el hospital de infectología “Dr. José Daniel Rodriguez Maridueña” en el periodo 2015-2016 Vol. 2, núm. 1., (2018)

Glubis Gomez Pelaez; Carlos Lara Zambrano; Hugo Luna Rodriguez; Carlos Lara Parra

Reporte de casos de VIH+ criptococosis de acuerdo a egreso hospitalario en el año 2016

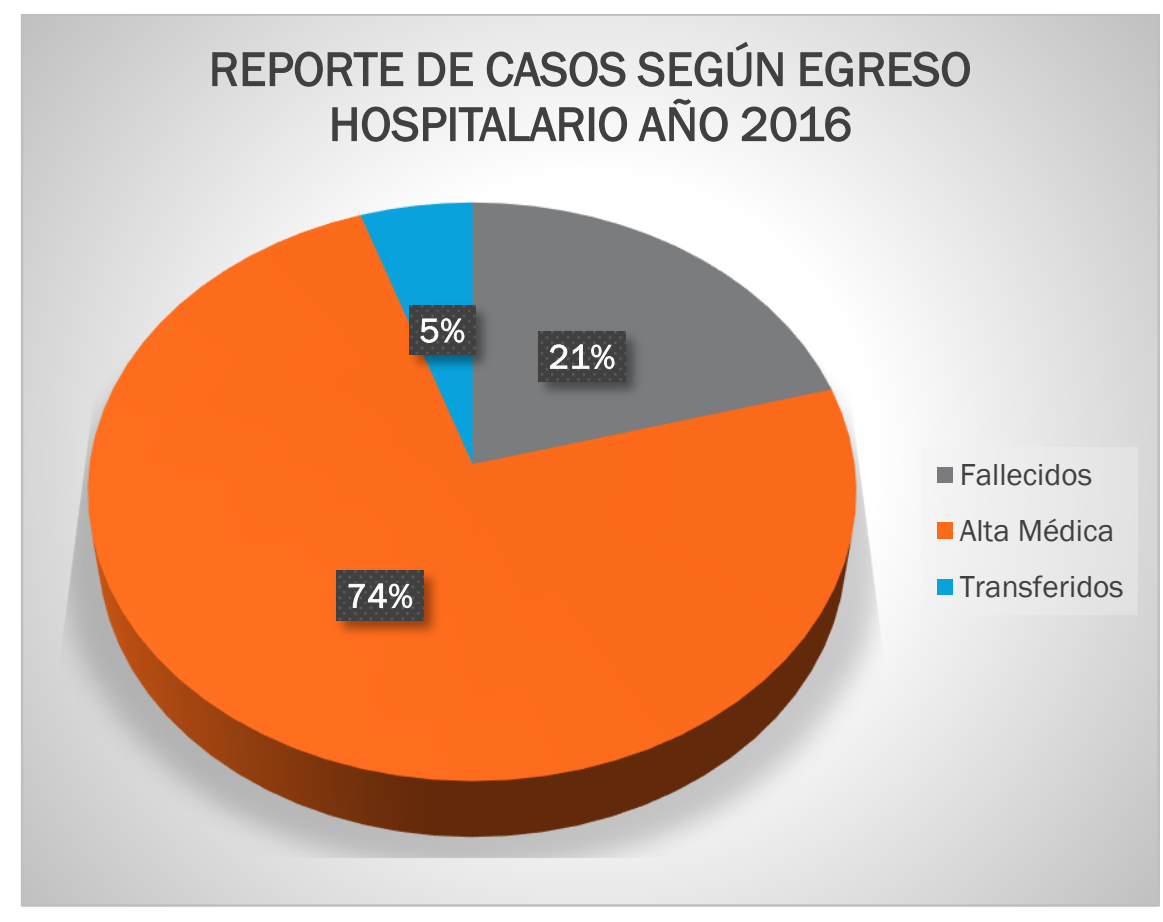

Figura $\mathrm{N}^{\circ}$ 8.- Casos de VIH+ criptococosis de acuerdo a egreso hospitalario en el 2016

Interpretación: En el año 2016 el porcentaje de fallecidos fue de $21 \%$ un poco menor al del año anterior.

\section{Conclusiones.}

El presente estudio ha tenido como objetivo determinar qué factores de riesgo predisponen al desarrollo de la infección por criptococo en pacientes VIH que ingresaron al Hospital de infectologia Dr. Daniel Rodríguez Maridueña, se encontró que la aparición de la enfermedad tiene asociación con el deficiente estado inmunológico, reflejado en el número de células CD4, evidenciándose en la mayoría de los pacientes una concentración en sangre <200cel/mm3 CD4. 


\section{Factores de riesgo de la Criptococosis en pacientes con VIH en el hospital de infectología "Dr. José Daniel Rodriguez Maridueña" en el periodo 2015-2016}

Vol. 2, núm. 1., (2018)

Glubis Gomez Pelaez; Carlos Lara Zambrano; Hugo Luna Rodriguez; Carlos Lara Parra

Se demostró además que la población rural es donde se presenta más comúnmente la criptococosis, lo que presupone el mayor contacto que tienen los habitantes de dicha zona con los nichos en que se desarrolla el criptococo.

La edad y el sexo no demostraron tener relación con la presentación de la micosis.

\section{Bibliografía.}

1. Padilla Desgarennes MdC, Navarrete Franco G, Pérez Cortés S. Criptococosis diseminada asociada con VIH. Dermatologia Revista Médica Mexicana. 2012 Oct; 53(2): p. 126-131.

2. Nucci M QTFCAyc. www.bago.com. [Online].; 2010. Available from: http://www.bago.com/BagoArg/Biblio/infectoweb585.htm

3. Lorena JM. Manifestaciones neurológicas del virus VIH. Rev. "Medicina" Vol. 15 N 2. 2010;: p. 139.

4. Tomás Baró MT. Epidemiologia de la Criptococosis en España. [Online].; 2012. Available from: http://www.tdx.cat/bitstream/handle/10803/3864/mtbt1de2.pdf?sequence=1.

5. Tircio Esparza SS. Determinación de criptococosis en pacientes con vih factores de riesgo y complicaciones en el hospital de infectologia "Dr Jose Daniel Rodriguez Maridueña" en el periodo 2013-2014. Trabajo de titulación presentado como requisito para optar por el grado de médico. Guayaquil: Universidad de Guayaquil, Facultad de Ciencias Médicas; 2015.

6. Torres M. Cuidado de la Salud. [Online].; 2014. Available from: http://www.cuidadodelasalud.com/salud/que-es-la-enfermedad-de-criptococosis/.

7. Tangarife C. Aprende en línea. [Online].; 2011. Available from: http://aprendeenlinea.udea.edu.co/lms/moodle/mod/page/view.php?id=100866.

8. Tello M, Gutiérrez E, Béjar V, Galarza C, Ramos W. Criptococosis. Rev. Méd. Risaralda. 2013 Sep; 19(2): p. 147-153.

9. Alan M, Sugar M. www.merckmanuals.com. [Online].; 2016. Available from: http://www.merckmanuals.com/es-pr/professional/enfermedadesinfecciosas/hongos/criptococosis.

10. Paúl SCD. Criptococosis en pacientes con SIDA: manifestaciones cutáneas y sistémicas, diagnóstico y terapéutica. Revista CES Medicina. 2010; 10(2): p. 79-89. 
Factores de riesgo de la Criptococosis en pacientes con VIH en el hospital de infectología “Dr. José Daniel Rodriguez Maridueña” en el periodo 2015-2016

Vol. 2, núm. 1., (2018)

Glubis Gomez Pelaez; Carlos Lara Zambrano; Hugo Luna Rodriguez; Carlos Lara Parra

11. Trombetta L. Manifestaciones clínicas de la criptococosis asociada al Sida. Revista Panamericana de Infectologia. 2010 Mar; 43(1): p. 13-16.

12. ADAM. CLINICA DAM. [Online].; 2016. Available from: https://webcache.googleusercontent.com/search?q=cache:Cgkw4UiG3ikJ:https://www.clinic adam.com/salud/5/001328.html+\&cd=8\&hl=es-419\&ct=clnk\&gl=ec. 